\title{
Case Report: A Young Man With Giant Pericardial Synovial Sarcoma
}

\author{
Yong Luo ${ }^{1}$, Ke Gong ${ }^{1}$, Ting Xie ${ }^{1}$, Ruilin Liu ${ }^{1}$, Hui Guo ${ }^{1}$, Lei Wang ${ }^{1}$, Zhiping Tan ${ }^{2}$, \\ Shi jun $\mathrm{Hu}^{1}$, Yifeng Yang ${ }^{1}$ and $\mathrm{Li} \mathrm{Xie}^{1 *}$ \\ ${ }^{1}$ Department of Cardiovascular Surgery, The Second Xiangya Hospital of Central South University, Central South University, \\ Changsha, China, ${ }^{2}$ The State Key Laboratory of Medical Genetics, The Clinical Center for Gene Diagnosis and Therapy, The \\ Second Xiangya Hospital of Central South University, Central South University, Changsha, China
}

Pericardial synovial sarcomas are sporadic tumors. Herein, we report a case of primary pericardial synovial sarcoma originating from the right pericardium. Missed diagnosis delayed surgical treatment. Eventually, the tumor occupied the almost entire pericardial cavity. The pericardial tumor was surgically removed as soon as possible after admission. In this paper, we aim to provide details that can help further understand the differing symptoms and presentations of pericardial synovial sarcoma and highlight the importance of consideration of this disease in similar cases where the etiology of pericardial effusion is unknown.

\section{OPEN ACCESS}

Edited by:

Robert Jeenchen Chen, Stanford University, United States

Reviewed by: Michael Hofmann, University of Zurich, Switzerland Paul Philipp Heinisch, German Heart, Germany

*Correspondence:

Specialty section:

This article was submitted to

Heart Surgery,

a section of the journal

Frontiers in Cardiovascular Medicine

Received: 05 December 2021 Accepted: 03 January 2022

Published: 27 January 2022

Citation:

Luo Y, Gong K, Xie T, Liu R, Guo H, Wang L, Tan Z, Hu Sj, Yang Y and Xie L (2022) Case Report: A Young Man With Giant Pericardial Synovial

doi: 10.3389/fCvm.2022.829328
Keywords: pericardial synovial sarcomas, pericardium, tumor, treatment, prognosis

\section{INTRODUCTION}

Pericardial synovial sarcoma (PSS) is a rare malignant tumor. Thus far, only a few cases of PSS have been reported. Synovial sarcoma (SS) is also reported in some uncommon sites such as the pleura, heart, kidney, lung, prostate, liver, gastrointestinal tract, and peripheral nerves. The diagnosis of SS in these unusual locations is challenging and requires additional diagnostic procedures such as immunohistochemistry, electron microscopy, and molecular genetic techniques for confirmation of the diagnosis. The case cardiac tamponade presented as the initial symptom, but no obvious evidence supported the diagnosis of pericardial synovial sarcoma. After 5 months, the anterior mediastinal tumor was found using transthoracic echocardiography. Non-random occurrence of $\mathrm{t}(\mathrm{x} ; 18)$ was consistently found in SS. In some cases, the only cytogenetic abnormality noted is the $t(X ; 18)$, suggesting that this is a key molecular event in tumor development (1). The survival rate of patients with primary cardiac sarcoma is poor, and most patients die within a few months after the diagnosis is confirmed. However, because of the rarity of PSS, it is difficult to determine the prognostic factors; however, diagnosis at a young age, lack of complicated chromosome abnormalities, and tumor originating from the pericardium appear to be factors pointing to good prognosis (2).

\section{CASE REPORT}

A 19-year-old man was referred to our hospital for the first time because of chest tightness and dyspnea. Chest echocardiography and computed tomography (CT) revealed a large amount of pericardial effusion, pleural effusion, left lung swelling, and right lower lung swelling (Figure 1A). The patient was subjected to pericardiocentesis and drainage; the drained fluid was sent for pathological examination. The pathology report revealed a lack of tumor cells (Figure 2A); acid-fast staining of the sample was also not positive for Mycobacterium tuberculosis (Figure 2B). The 

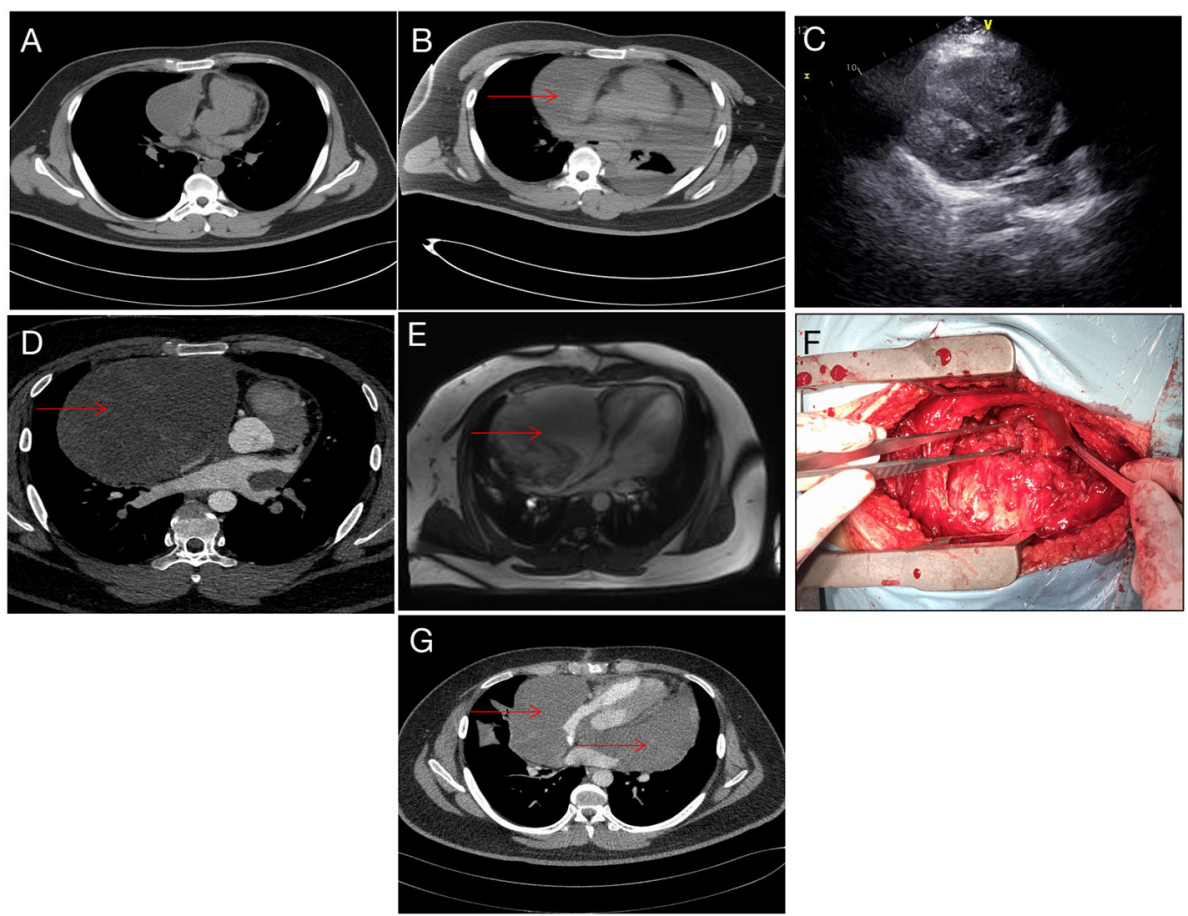

FIGURE 1 | Imaging data. (A) CT image taken during the first admission. (B) After pericardiocentesis, CT indicating a suspicious mass (red arrow).

(C) Echocardiography showing pericardial space-occupying lesions. (D) Reexamination after 5 months showed obvious space occupation (red arrow).

(E) Preoperative magnetic resonance imaging, right pericardium, showing a cystic mass (red arrow). (F) During the operation, the tumor filled the pericardial cavity. (G) Tumor recurrence noted 5 months after operation (red arrow).
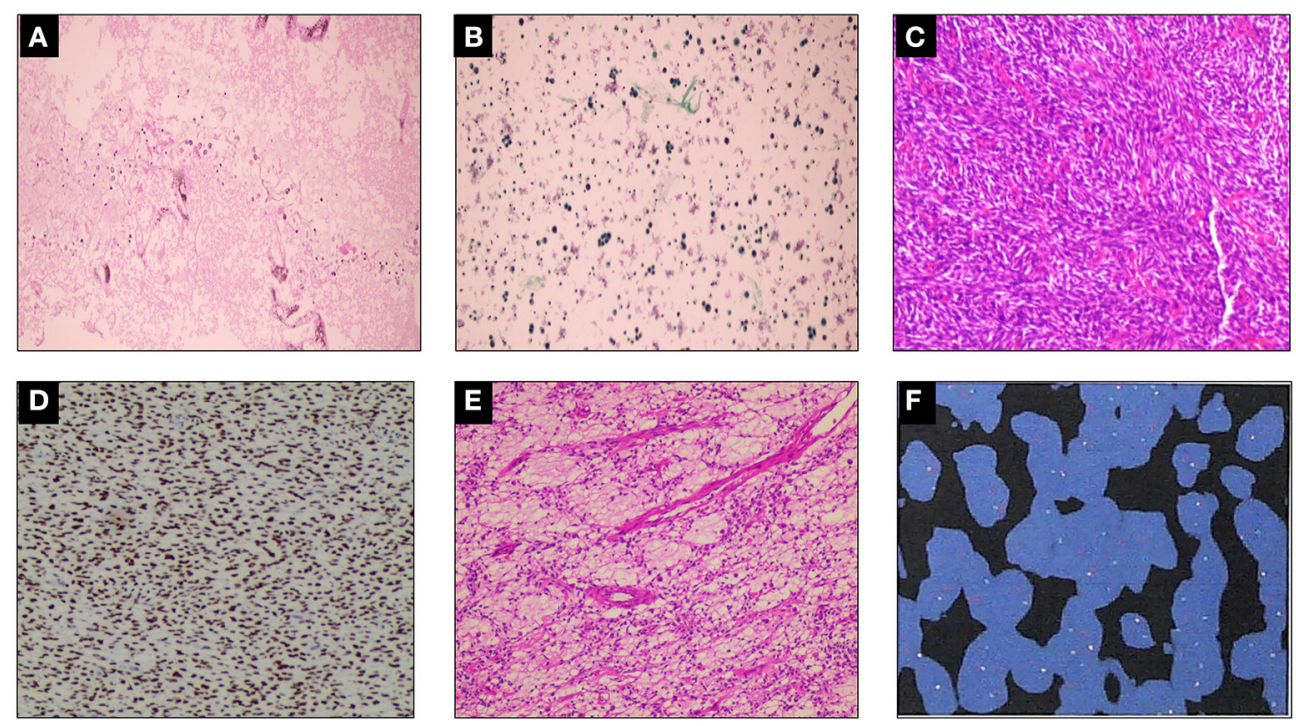

FIGURE 2 | Histopathological examination. (A) A small number of mesothelial cells and lymphocytes were found in the pleural effusion, but no malignant tumor cells were found. (B) Tissue cells, lymphocytes, and no malignant tumor cells could be found in the left pleural effusion. Mycobacterium tuberculosis was found to be negative by acid-fast staining (-). (C-E) Damaged tissue of pericardial tumor, which measured $20 \times 17 \times 5 \mathrm{~cm}$, with some gelatinous areas. Mesenchymal tissue-derived tumors, with dense spindle cell areas and edema areas, combined with immunohistochemistry, do not rule out SS. Immunohistochemistry results: CK $(-)$, EMA (focal +), TLEL (+), CD34 (-), S100 (-), CD99 (partial +), Bcl-2 (+), Ki67 (hot spot 25\% +), WT-1 (-), Desmin (-), SMA (-), Vim (+), calponin (partial +), INl-1 $(-)$, ERG (-), p53 (30\% weak +), HMB45 (-), FLI-1 (-). (F) Fluorescence in situ hybridization (FISH) showing rearrangement of the SS18 gene, indicating positive result. 
patient was also subjected to bone marrow puncture; however, the biopsy sample did not show any positive results. Following pericardiocentesis and treatment aimed at providing symptomatic relief, his pleural effusion and pericardial effusion were absorbed (Figure 1B). He was then discharged after symptom improvement. Therefore, some special manifestations of patients have been ignored. Although a mediastinal mass was noted in the first CT images, the pathological examination and related auxiliary examinations of pericardial effusion did not discover any positive results (Figure 1B). Five months later, the patient was re-examined at the local hospital and was found to have a pericardial mass through echocardiography and chest CT.

Then the patient had seen a doctor again for further treatment. Echocardiography, CT, and nuclear magnetic resonance imaging (MRI) at our hospital indicated that the right anterior mediastinum mass had compressed the right heart (Figure 1C) and the aortic root; it measured $15.3 \times 11.7 \times 15.1 \mathrm{~cm}$ (Figures 1D,E). He had no family history relevant to cardiac complaints. Following relevant preoperative examinations, he underwent mass resection via a median sternum incision. The size of the mass was about $20 \times 15 \times 19 \mathrm{~cm}$ (Figure 1F). The mass was found to completely cover the right atrium, superior and inferior vena cava, ascending aorta, and part of the pulmonary artery; it also partly covered the anterior wall of the right ventricle. After the operation, pathological examination (Figures 2C-E); furthermore, an SS18 gene test was done (Figure 2F). It was found that the SS18 gene was broken, so the result was positive, suggesting SS. A postoperative echocardiography performed 2 months after discharge demonstrated a heterogeneous hypoechoic lump in the pericardium; thus, tumor recurrence was considered. After 2 months of treatment with traditional Chinese medicine, he was referred to our hospital again. CT angiography revealed a more significant layer of cystic consolidation on the left margin of the heart that measured about $12 \times 6.9 \mathrm{~cm}$ (left), $11.5 \times 5.6 \mathrm{~cm}$ (right) (Figure 1G). Thus, following discussion with the patient, chemotherapy was initiated. The patient is currently undergoing chemotherapy with regimen at the oncology department of our hospital.

\section{DISCUSSION}

The incidence of heart tumors is quite hidden, and most of them are caused by accidentally discovered cardiac lumps. These occasional phenomena often means thrombus or neoplasms, which usually occur in specific clinical environments (3). The incidence rate of cardiac tumors ranges from 0.001 to $0.03 \%$. The overwhelming majority of these tumors are benign, and only about one-fourth are malignant. PSS is an even rarer form of cardiac tumors. As of 2018, only 36 cases have been reported in the literature; thus, limited data prevent further understanding of this condition (4). However, this lesion undoubtedly predicts a dismal prognosis (5).

SS is an uncommon malignant tumor mainly seen in children and adolescents; its 10-year overall survival rate is about 0 $20 \%(6,7)$. The name SS is possibly indicative of its origin in synovial tissue. It has a biphasic appearance on histology and is divided into two different subtypes: spindle and epithelial cell subtypes. However, no epithelioid differentiation was found in tumor tissues, neither were epithelial markers, such as IHC staining of cytokeratin, observed. Therefore, the real origin of SS remains unknown. The characteristic chromosomal abnormality of SS is $\mathrm{t}(\mathrm{X} ; 18)(\mathrm{p} 11.2 ; \mathrm{q} 11.2)$. This has a sensitivity of about $90-100 \%$, strongly suggesting its direct involvement in certain aspects of the occurrence and progression of this cancer $(1,8)$. Even in our case, the diagnosis of SS was proven following a positive result of the SS18 genetic testing.

The morbidity rate of young people with PSS is relatively high, and the most common site of the disease is in the pericardium (9). Even in our case, the tumor originated from the pericardium. The clinical symptoms of the disease are non-specific, with dyspnea and chest pain being the most common presenting symptoms. The patient described herein presented with chest tightness, dyspnea, and fatigue before admission. There was a large amount of pericardial effusion noted in the early stage. According to a new study conducted in China, the most common causes of pericardial effusion were found to be malignant tumors and tuberculosis (10). However, in our case, pericardiocentesis and etiological examination of the pleural effusion did not support the diagnosis of malignant pleural effusion. This could be possible because malignant tumor cells may not be present in $25 \%$ of the cases of malignant pericardial effusion (10). Therefore, despite the absence of malignant cells in the pericardial effusion, the possibility of malignant pleural effusion should not be completely ruled out.

The reason for the initially missed diagnosis in our case was the absence of tumor cells and related markers in the pleural fluid. Besides, the patient's symptoms improved significantly after symptomatic treatment. However, the initial beginning stage of the tumor can be seen on the CT performed initially. After 5 months, the tumor had grown up, leading to aggravation of the patient's condition. It was visible on echocardiography and compressed the right heart and the root of the great artery.

As regards the treatment of PSS, on the one hand, due to the invasiveness and expansibility of the tumor, it is generally impossible to remove the tumor completely. On the other hand, there is no systemic study on this topic due to extremely low incidence of the disease; thus, no optimal treatment strategy has yet been proposed. Besides surgical resection, radiotherapy and chemotherapy are also used; this, however, depends on the clinician's discretion and the patient's condition. In general, surgical resection should be performed as soon as possible once the tumor diagnosis is confirmed, especially when the possibility of malignancy is high. Resection can be performed based on the preoperative MRI evaluation, with the premise of negative margin. Because positive margin is an independent risk factor for cardiac tumors, we recommend MRI before surgery to evaluate the degree of disease and assess whether it can be resected on the premise of negative margin. If this is not possible, second-line radiotherapy and chemotherapy can be considered; evaluation can then be performed and resection undertaken after the tumor has shrunk in size until it can be resected with a negative margin (11). Reports of successful 
total artificial heart transplantation have been published, and this can also be a solution (12). Although patients with PSS have a low probability of lung metastasis, according to the evaluation by Lee et al., metastatic resection is a good choice for metastatic SS (13). In a recent study, a comparative result of the treatment of primary cardiac sarcoma was reported. Although some authors found no improvement in survival rate compared with traditional surgical treatment $(14,15)$. However, other authors have found that many patients survive longer after transplantation (16). The patient described herein also considered heart transplantation but considered chemotherapy first due to the lack of donors.

As regards prognosis, a recent statistical study showed that the 2-year survival rate following complete surgical resection of PSS was $75.2 \%$ ( \pm 9.7 SE), while that following incomplete resection was $55.0 \%( \pm 11.5 \mathrm{SE})$ (Log-Rank test: $P$ $=0.029)$. The survival rate at 12 months was $96.5 \%( \pm 3.8$ $\mathrm{SE})$ in patients treated with chemoradiotherapy (CRT), while it was $21.9 \%( \pm 10.8 \mathrm{SE})$ in those not treated with CRT (Log-Rank test: $P \leq 0.0001$ ) (17). Thus, it was concluded that advanced age and absence of CRT were significantly associated with reduced survival, while total tumor resection was associated with remarkably improved survival. Furthermore, the clinical outcomes of patients receiving postoperative adjuvant chemotherapy was good, and adjuvant chemotherapy was found to be associated with significantly prolonged survival (1822). Putnam et al. showed that radiotherapy alone seemed to have little impact on prognosis (23). The age of patients with PSS was also shown to be a strong prognostic indicator in this study. In a large meta-analysis of 1,268 patients with SS, Sultan et al. found that younger patients had better outcomes than older patients; (24) this finding was also confirmed by other authors (25-27). The patient in our report was 19 years old; thus, this young age is indicative of a favorable prognosis. After surgical resection, the patient relapsed 2 months later and was transferred to the Department of Oncology for chemotherapy.

\section{REFERENCES}

1. Sandberg AA, Bridge JA. Updates on the cytogenetics and molecular genetics of bone and soft tissue tumors. Synovial sarcoma Cancer Genet Cytogenet. (2002) 133:1-23. doi: 10.1016/s0165-4608(01)00626-4

2. Varma T, Adegboyega P. Primary cardiac synovial sarcoma. Arch Pathol Lab Med. (2012) 136:454-8. doi: 10.5858/arpa.2011-0008-RS

3. Bruce CJ. Cardiac tumours: diagnosis and management. Heart. (2011) 97:15160. doi: 10.1136/hrt.2009.186320

4. Duran-Moreno J, Kampoli K, Kapetanakis EI, Mademli M, Koufopouos $\mathrm{N}$, et al. Pericardial synovial sarcoma: case report, literature review and pooled analysis. In Vivo. (2019) 33:1531-8. doi: 10.21873/invivo. 11633

5. Ouarrak S, El Ouali Z, Elkebir A, Moumna K, Karkouri M, Azzouzi L, et al. Diagnostic approach to a cardiac mass: a case report of misdiagnosed cardiac synovial sarcoma. Eur Heart J Case Rep. (2021) 5:ytab039. doi: 10.1093/ehjcr/ytab039

6. Boulmay B, Cooper G, Reith JD, Marsh R. Primary cardiac synovial sarcoma: a case report and brief review of the literature. Sarcoma. (2007) 2007:94797. doi: 10.1155/2007/94797

\section{CONCLUSION}

Herein, we describe a case of a patient with PSS. The missed diagnosis highlights the necessity and difficulty of differential diagnosis in patients with such tumors. Histopathology is key to the diagnosis of PSS. In our case, no abnormality in the pericardial effusion was noted, and our patient presented with atypical clinical symptoms. Although primary PSS is not common, when the etiological diagnosis of pericardial effusion is unknown, further tests need to be carried out to help rule out or confirm the diagnosis of PSS.

\section{DATA AVAILABILITY STATEMENT}

The raw data supporting the conclusions of this article will be made available by the authors, without undue reservation.

\section{ETHICS STATEMENT}

Written informed consent was obtained from the individual(s) for the publication of any potentially identifiable images or data included in this article.

\section{AUTHOR CONTRIBUTIONS}

YL, ZT, and LX contributed to conception and design of the study. YL completed the data collection and wrote the first draft of the manuscript. YL, KG, TX, HG, RL, SH, and LW wrote sections of the manuscript. All authors contributed to manuscript revision, read, and approved the submitted version.

\section{FUNDING}

This study was supported by the National Science Foundation for Young Scientists of China (8150020951) and the Natural Science Foundation for Young Scientists of Hunan Province (2016JJ4099).

7. Coli A, Chiariello GA, Novello M, Colizzi C, Massetti M. Treatment of cardiac synovial sarcoma: experience of two cases. J Cardiothorac Surg. (2018) 13:84. doi: 10.1186/s13019-018-0771-0

8. Kawai A, Woodruff J, Healey JH, Brennan MF, Antonescu CR, Ladanyi M. SYT-SSX gene fusion as a determinant of morphology and prognosis in synovial sarcoma. $N$ Engl J Med. (1998) 338:153-60. doi: 10.1056/NEJM199801153380303

9. Coindre JM. New WHO classification of tumours of soft tissue and bone. Ann Pathol. (2012) 32 Supplement:S115-6. doi: 10.1016/j.annpat.2012. 07.006

10. Ma W, Liu J, Yeng Z, Chen S, Zheng Y, Ye S, et al. Causes of moderate to large pericardial effusion requiring pericardiocentesis in 140 Han Chinese patients. Herz. (2012) 37:183-7. doi: 10.1007/s00059-0113428-5

11. Kim MP, Correa AM, Blackmon S, Quiroga-Garza G, Weilbaecher D, Bruckner B. et al. Outcomes after right-side heart sarcoma resection. Ann Thorac Surg. (2011) 91:770-6. doi: 10.1016/j.athoracsur.2010.09.079

12. Bruckner BA, Abu Saleh WK, Al Jabbari O, Copeland JG, Estep JD, Loebe M. Total artificial heart implantation after excision of right ventricular angiosarcoma. Tex Heart Inst J. (2016) 43:252-4. doi: 10.14503/THIJ-15-5131 
13. Lee K, Chul Kang M, Lee HW, Park JH, Baek HJ, Cho SJ, et al. Pulmonary metastasectomy in adult patients with synovial sarcoma: a single-center experience. Korean J Thorac Cardiovasc Surg. (2016) 49:4515. doi: 10.5090/kjtcs.2016.49.6.451

14. Michler RE, Goldstein DJ. Treatment of cardiac tumors by orthotopic cardiac transplantation. Semin Oncol. (1997) 24:534-9

15. Jiménez Mazuecos JM, Manso RF, Cubero JS, Ramos JT, Oteo Dominiguez JF. Rivera LAP Is heart transplantation for primary cardiac sarcoma a useful therapeutic option? Rev Esp Cardiol. (2003) 56:408-11. doi: 10.1016/s0300-8932(03)76886-9

16. Gowdamarajan A, Michler RE. Therapy for primary cardiac tumors: is there a role for heart transplantation? Curr Opin Cardiol. (2000) 15:1215. doi: 10.1097/00001573-200003000-00010

17. Coli A, Cassano A, Novello M, Ranelletti FO, Lauriola L. Primary cardiac synovial sarcoma: a review correlating outcomes with surgery and adjuvant therapy. J Card Surg. (2019) 34:1321-7. doi: 10.1111/jocs. 14214

18. Kim CH, Dancer JY, Coffey D, Zhai QJ, Reardon M, Ayala AG, et al. Clinicopathologic study of 24 patients with primary cardiac sarcomas: a 10-year single institution experience. Hum Pathol. (2008) 39:9338. doi: 10.1016/j.humpath.2007.12.018

19. Look Hong NJ, Pandalai PK, Hornick JL, Shekar PS, Harmon DC, Chen YL, et al. Cardiac angiosarcoma management and outcomes: 20-year single-institution experience. Ann Surg Oncol. (2012) 19:270715. doi: 10.1245/s10434-012-2334-2

20. Mayer F, Aebert H, Rudert M, Konigsrainer A, Horger M, Kanz L, et al. Primary malignant sarcomas of the heart and great vessels in adult patients-a single-center experience. Oncologist. (2007) 12:113442. doi: 10.1634/theoncologist.12-9-1134

21. Eckstein R, Gössner W, Rienmüller R. Primary malignant fibrous histiocytoma of the left atrium. Surgical and chemotherapeutic management. Br Heart J. (1984) 52:354-7. doi: 10.1136/hrt.52.3.354

22. Aboud A, Farha K, Hsieh WC, Brasch F, Esminger S, Gummert J, et al. Prognostic factors for long-term survival after surgical resection of primary cardiac sarcoma. Thorac Cardiovasc Surg. (2019) 67:66571. doi: 10.1055/s-0039-1692409
23. Putnam JB Jr., Sweeney MS, Colon R, Lanza LA, Frazier OH, Cooley DA. Primary cardiac sarcomas. Ann Thorac Surg. (1991) 51:906-10. doi: 10.1016/0003-4975(91)91003-e

24. Sultan I, Rodriguez-Galindo C, Saab R, Yasir S, Casanova M, Ferrari A. Comparing children and adults with synovial sarcoma in the Surveillance, Epidemiology, and End Results Program, 1983 to 2005: an analysis of 1268 patients. Cancer. (2009) 115:3537-47. doi: 10.1002/cncr.24424

25. Bergh P, Meis-Kindblom JM, Gherlinzoni F, Berlin O, Bacchini P, Bertoni F, et al. Synovial sarcoma: identification of low and high risk groups. Cancer. (1999) 85:2596-607. doi: 10.1002/(sici)1097-0142(19990615)85:12<2596::aidcncr16>3.0.co; $2-\mathrm{k}$

26. Spurrell EL, Fisher C, Thomas JM, Judson IR. Prognostic factors in advanced synovial sarcoma: an analysis of 104 patients treated at the Royal Marsden Hospital. Ann Oncol. (2005) 16:437-44. doi: 10.1093/annonc/mdi082

27. Vlenterie M, Ho VKY, Kaal SEJ, Vlenterie R, Haas R, van der Graaf WTA. Age as an independent prognostic factor for survival of localised synovial sarcoma patients. Br J Cancer. (2015) 113:1602-6. doi: 10.1038/bjc.2015.375

Conflict of Interest: The authors declare that the research was conducted in the absence of any commercial or financial relationships that could be construed as a potential conflict of interest.

Publisher's Note: All claims expressed in this article are solely those of the authors and do not necessarily represent those of their affiliated organizations, or those of the publisher, the editors and the reviewers. Any product that may be evaluated in this article, or claim that may be made by its manufacturer, is not guaranteed or endorsed by the publisher.

Copyright @ 2022 Luo, Gong, Xie, Liu, Guo, Wang, Tan, Hu, Yang and Xie. This is an open-access article distributed under the terms of the Creative Commons Attribution License (CC BY). The use, distribution or reproduction in other forums is permitted, provided the original author(s) and the copyright owner(s) are credited and that the original publication in this journal is cited, in accordance with accepted academic practice. No use, distribution or reproduction is permitted which does not comply with these terms. 